\title{
Properties of the effective noise in the nonlinear Fourier transform-based transmission
}

\author{
Maryna Pankratova $^{1 *}$, Anastasiia Vasylchenkova ${ }^{1 *}$, Jaroslaw E. Prilepsky $^{1}$, \\ Stanislav A. Derevyanko ${ }^{2}$ \\ ${ }^{1}$ Aston Institute of Photonic Technologies, Aston University, Birmingham B4 7ET, UK \\ ${ }^{2}$ Department of Electrical and Computer Engineering, Ben-Gurion University of the Negev, Beer Sheva 84105, Israel \\ m.pankratova@aston.ac.uk \\ * These two authors contributed equally to this work
}

\begin{abstract}
We investigate the correlation properties of optical noise in nonlinear Fourier domain for communication systems using the nonlinear Fourier transform. Effective covariance functions are obtained numerically and compared with theoretical predictions.
\end{abstract}

OCIS codes: $070.4340,060.2330$.

\section{Introduction}

Optical transmission based on nonlinear Fourier transform (NFT) has attracted a lot of attention recently as a promising tool for nonlinearity mitigation [1]. The NFT can be applied to the so-called integrable systems, to which belongs the the nonlinear Schrödinger equation (NLSE), i.e. the master model governing the propagation of optical field envelope function $[1,2]$. For modelling the signal propagation down the single-mode optical fibre, in our current work we assume ideal distributed amplification but account for the amplifier spontaneous emission. As the data carrier, in this work we use only the continuous NFT spectrum $r(\xi)$ (the complex valued function of the real nonlinear "frequency" $\xi$ ), utilising the nonlinear inverse synthesis (NIS) signal modulation method; the system scheme and details of the approach can be found in $[2,3]$. For the NIS we use the orthogonal frequency division multiplexing (OFDM) modulation of the spectral data with 128 QPSK-modulated subcarriers in each OFDM symbol (the transmission takes place in the burst mode to prevent the inter-symbol interference). The accumulated noise inside the NFT domain after propagation over distance $L$ can be modelled as an effective additive term $N(\xi, r(\xi, 0))$, defined via the equalized (i.e. compensated) propagation inside the NF domain [2]: $e^{-4 \xi^{2} L} r(\xi, L)=r(\xi, 0)+N(\xi, r(\xi, 0))$. Understanding the properties of this noise is crucial for (i) transmission optimisation and noise mitigation, and (ii) for the channel capacity evaluation.

One important feature of the noise $N(\xi, r(\xi, 0))$ is that it is input-dependent. Also the two quadratures of the noise are not symmetric, which means that apart from the covariance function $\mathbb{E}\left[N(\xi) N^{*}\left(\xi^{\prime}\right)\right]$ one must also consider a pseudocovariance $\mathbb{E}\left[N(\xi) N\left(\xi^{\prime}\right)\right]$. In [2] the asymptotic expressions for covariance and pseudo-covariance of this effective noise assuming ideal distributed amplification, high signal-to-noise ratio (SNR) and sufficiently long distances were derived. The resulting expression are as follows:

$$
\begin{gathered}
\mathbb{E}\left[N(\xi) N^{*}\left(\xi^{\prime}\right)\right]=2 D L \pi \delta\left(\xi-\xi^{\prime}\right) E_{1}(\xi), \quad E_{1}(\xi)=1+|r(\xi, 0)|^{2}+|r(\xi, 0)|^{4}, \\
\mathbb{E}\left[N(\xi) N\left(\xi^{\prime}\right)\right]=2 D L \pi \delta\left(\xi-\xi^{\prime}\right) E_{2}(\xi), \quad E_{2}(\xi)=r(\xi, 0)^{2} .
\end{gathered}
$$

where $D$ is the normalised power spectral density of noise in optical domain. The expressions above apply for any $r(\xi)$ modulation format. For the capacity estimation we need to average the expression above over initial realisations. For the case of the Gaussian distribution of coefficients, the analytical formula for the correlator functions averaged over the initial coefficients distribution was obtained in [2] which in our notations reads:

$$
\left\langle E_{1}(\xi)\right\rangle=1+S N_{b}\left(T_{s} / 2 \pi\right)+2 S^{2} N_{b}^{2}\left(T_{s} / 2 \pi\right)^{2}
$$

where $S=\left\langle\left|c_{k}\right|^{2}\right\rangle, c_{k}$ are the data-carrying input coefficient, $T_{s}$ is the symbol temporal width, $N_{b}$ is number of OFDM symbols and $\langle\ldots\rangle$ means the average over initial distribution.

In this work we numerically study the properties of effective nonlinear noise arising inside the NFT domain due to the progenitor amplification-induced noise in the space-time domain. Our goal is to compare the numerical simulation results with the analytical asymptotic expressions (3), first derived in [2], to understand how close the asymptotic expression to the true picture and how accurate the estimations presented in Ref. [2] are. 


\section{Results}

We simulate the NIS transmission of optical signal with the temporal support $12 \mathrm{~ns}$ down the single-mode fibre with standard parameters [2-4] for propagation distance of $1000 \mathrm{~km}$. Each NIS-modulated time-domain signal is a burst padded with zero wings; the duration of the latter is equal to the dispersion-induced memory. In Fig. 1 we present the plots depicting the numerical data for the absolute value of diagonal elements $\left(\xi=\xi^{\prime}\right)$ of the covariance $\left|\mathbb{E}\left[N(\xi) N^{*}\left(\xi^{\prime}\right)\right]\right|$ and pseudocovariance $\left|\mathbb{E}\left[N(\xi) N\left(\xi^{\prime}\right)\right]\right|$ as a function of nonlinear "frequency" $\xi$. Each point in this figure was obtained by averaging over 100 realizations of in-line noise and over 600 realizations of 128 QPSKmodulated subcarriers. Signal power levels were chosen in the vicinity of the optimal $\mathrm{Q}^{2}$-factor value (not shown).

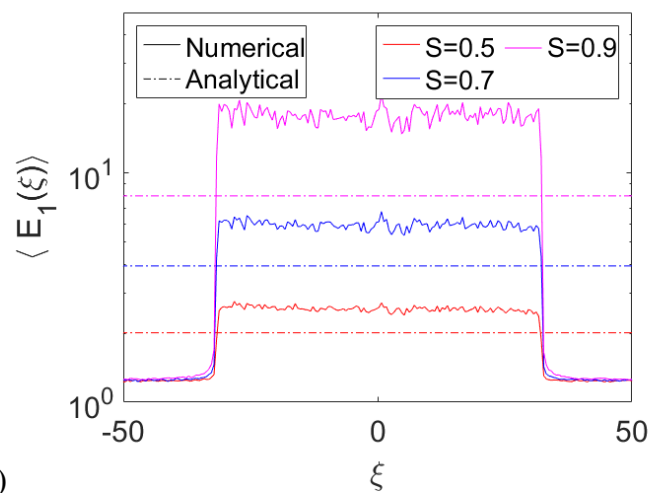

(a)

Fig. 1. The diagonal elements $\left(\xi=\xi^{\prime}\right)$ of the covariance close to optimal point, in comparison with analytical results [2]).

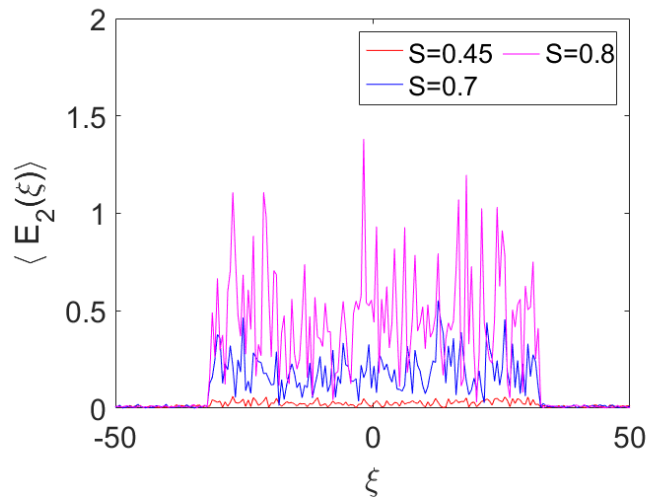

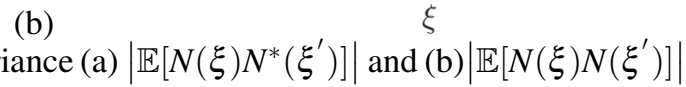

It can be seen from Fig. 1 that a satisfactory agreement with the results from [2] (dashed lines) is observed aside from very high powers case (magenta solid line in Fig.1). The difference between the analytical predictions and our data at large power levels can be attributed to higher order effects not accounted for by the simplified asymptotic theory of Ref. [2] and to non-adiabatic effects such as the creation of spurious discrete eigenvalues (solitons) from noise. The latter issue however requires further study.

\section{Conclusions}

The nonlinear spectral noise in the NIS-based optical transmission systems, possesses several non-trivial properties. We observed the qualitative agreement with the theoretical predictions: both covariance and pseudocovariance are input-dependent, and the noise is non-circular. Some deviation of our numerical results from the asymptotic theory are caused by higher-order terms non-adiabatic effects and different probability distribution of the coefficients. Therefore, the noise affecting the NFT domain signal is larger then theoretically predicted one, what should be taken into account during the developing of NFT-based communication formats. The latter is important for deriving more precise capacity estimations for NFT-based transmission methods.

This project has received funding from the European Union's Horizon 2020 research and innovation programme under the Marie Sklodowska-Curie Grant Agreement No.751561.

\section{References}

1. S. K. Turitsyn, et al, "Nonlinear Fourier transform for optical data processing and transmission: advances and perspectives," Optica, Vol. 4, no.3 p. 307 (2017).

2. S. A. Derevyanko, J. E. Prilepsky, and S. K. Turitsyn, "Capacity estimates for optical transmission based on the nonlinear Fourier transform," Nat. Commun., Vol. 7, Art. No. 12710 (2016).

3. S. T. Le, J. E. Prilepsky, and S. K. Turitsyn, "Nonlinear inverse synthesis for high spectral efficiency transmission in optical fibers." Opt. Express, Vol. 22, p. 26720 (2014).

4. V. Aref, S.T. Le, and H. Buelow, "Modulation over Nonlinear Fourier Spectrum: Continuous and Discrete Spectrum”, J. Lightwave Technol., Vol. 36, no. 6, p. 1289 (2018). 